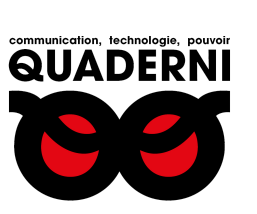

\title{
Quaderni
}

Communication, technologies, pouvoir

80 | Hiver 2012-2013

L'intégration européenne

\section{Enseigner les méthodes en sciences sociales : de quoi parle-t-on?}

\section{Sophie Duchesne}

\section{(2) OpenEdition \\ 12 Journals}

\section{Édition électronique}

URL : https://journals.openedition.org/quaderni/684

DOI : 10.4000/quaderni.684

ISSN : 2105-2956

Éditeur

Les éditions de la Maison des sciences de l'Homme

\section{Édition imprimée}

Date de publication : 5 janvier 2013

Pagination : 131-136

ISBN : 978-2-7351-1525-9

\section{Référence électronique}

Sophie Duchesne, «Enseigner les méthodes en sciences sociales : de quoi parle-t-on ? », Quaderni [En ligne], 80 | Hiver 2012-2013, mis en ligne le 05 janvier 2015, consulté le 21 septembre 2021. URL http://journals.openedition.org/quaderni/684 ; DOI : https://doi.org/10.4000/quaderni.684 


\section{Lecture croisée}

\section{Enseigner les méthodes en sciences sociales : de quoi parle-t-on?}

par Sophie Duchesne CNRS

ISP / Université Paris Ouest Nanterre

Enquêtes qualitatives, enquêtes quantitatives.

Pierre Bréchon (dir.)

Presses universitaires de Grenoble, 2011

\section{Manuel de recherche en sciences sociales.}

Luc Van Campenhoudt et Raymond Quivy (en coll. avec Jacques Marquet)

Dunod, 4e édition, 2011

Enquêtes qualitatives, enquêtes quantitatives vise à transmettre aux étudiants et autres (jeunes) chercheurs les techniques de réalisation d'une enquête par entretiens ou par questionnaires. Il s'appuie sur la longue expérience que les enseignants de Sciences Po Grenoble ont accumulée en matière d'enseignement des méthodes. Les auteurs (S. Abrial, J.-P. Bozonnet, P. Bréchon, F. Gonthier, S. Louvel et V. Tournier) sont tous membres de l'équipe PACTE et interviennent, pour la plupart, au sein du master PROGIS qui forme aux professions de l'étude d'opinion et de marché. Le ton est celui des travaux pratiques, volontairement clair, précis et concret, et conjugue le bon sens et l'expérience. Chacun des chapitres correspond à une série de cours qui couvrent toutes les étapes de réalisation de ces types d'enquêtes (construction de l'échantillon, élaboration des questions, passation et analyses) en passant d'abord en revue les techniques liées à l'usage de l'entretien puis celles du questionnaire. On soulignera l'effort fait, dans les deux cas, pour tenter de 
rendre compte des règles de l'analyse. Les exemples des chapitres introductifs sont pris dans la littérature canonique de sciences sociales mais les autres renvoient aussi à des enquêtes qui ont été réalisées autour des enseignements de l'équipe. Chaque chapitre s'accompagne d'exercices d'application dont les solutions sont mises en ligne sur un site compagnon (www.enquetesqualiquanti.fr). Le livre juxtapose donc deux méthodes d'enquête, par entretien et par questionnaire, avec la volonté affichée de dépasser ce que les auteurs considèrent comme une fausse opposition entre qualitatif et quantitatif, et d'œuvrer dans le sens de leur complémentarité. L'entretien est présenté par P. Bréchon comme l'outil privilégié d'une posture subjectiviste ou compréhensive, et le questionnaire, comme un outil au service d'une approche objectivante. Si les moyens le permettent, c'est la combinaison des deux approches et donc des deux ensembles de techniques qui conduiraient à faire vraiment le tour d'une question.

Si le livre répond à ses objectifs et propose aux futurs professionnels de l'enquête d'opinion des règles et des conseils clairs et utiles, son apport pour des jeunes chercheurs est plus discutable. La notion de « bonnes pratiques » en matière de méthodes d'enquête convient mieux aux approches routinisées qui sont celles des enquêtes appliquées qu'à la recherche en sciences sociales où la notion même de méthodes fait débat : savoir si l'on peut traiter de façon autonome la question des techniques mises en œuvre au cours d'une recherche et s'il existe un répertoire de règles «prêtes à l'emploi » dans lequel on peut puiser ne fait pas consensus, notamment en France. Le livre traite l'enquête par entretiens et l'enquête par questionnaires comme deux ensembles de techniques qu'on pourrait apprendre presque indépendamment de la démarche de recherche dans laquelle on les appliquerait. Ce choix prête le flanc à la critique de ceux qui voient dans la collection et le traitement des informations des moments indissociables de l'ensemble du processus de recherche. Et ce d'autant plus que l'ouvrage ne propose pas les références qui permettraient d'ouvrir des perspectives critiques sur le discours proposé : la bibliographie est de fait singulièrement limitée.

On s'étonne en particulier que le livre de Raymond Quivy et Luc van Campenhoudt, le Manuel de recherche en sciences sociales, dont la quatrième édition est parue au même moment, ne soit même pas cité. Ce manuel, dont la première édition date de 1988, est très largement utilisé dans les institutions universitaires de langue française sinon en France. Ses auteurs sont 
enseignants à l'Université Catholique de Louvain. La quatrième édition est la première depuis le décès de Raymond Quivy, et Luc Van Campenhoudt a trouvé en Jacques Marquet, sociologue comme lui, un collaborateur prêt à continuer l'aventure. Ce manuel s'inspire aussi très largement de l'expérience d'enseignants de ses auteurs et assume clairement un positionnement empirique qui fait de l'administration de la preuve l'objectif de la recherche en sciences sociales. Pour autant, le choix et l'application des méthodes ne constituent que des étapes parmi d'autres dont les plus importantes, voire les plus difficiles, sont du côté de la construction de la problématique et du modèle d'analyse. Au total, la présentation de la palette de méthodes de recueil et d'analyse des informations représente à peine une quarantaine de pages dans un volume qui en compte plus de 250 , auxquelles il faut ajouter des pages très bien venues, très concrètes elles aussi, sur l'étape dite d'exploration, dans laquelle le recours à des entretiens préliminaires est vivement recommandée et soigneusement explicitée.

Le livre mobilise de nombreux exemples, exposés en termes pédagogiques, et destinés notamment à faire valoir la variété des approches et des recherches en sciences sociales. Il ne cache pas l'existence de controverses qui gouvernent les choix, notamment méthodologiques, au-delà de l'opposition quali/ quanti. Il distingue sept grandes étapes de la démarche scientifique appliquée à nos disciplines : formuler la question de départ ; explorer le domaine de recherche ; formuler la problématique ; construire le modèle d'analyse ; concevoir et mettre en œuvre l'observation ; analyser les informations recueillies et écrire les conclusions. Chacune de ces étapes fait l'objet d'un chapitre détaillé, mais tout en faisant apparaître la limite des frontières entre ces étapes et les effets rétroactifs qui peuvent amener le chercheur à revenir sur des étapes antérieures au fil de la recherche. Vers la fin de l'ouvrage, les auteurs évoquent d'ailleurs en quelques pages ce qu'ils désignent par « field research » (autrement dit, la démarche ethnographique utilisée au-delà de l'anthropologie, sur le modèle développé par l'Ecole de Chicago) pour souligner qu'alors la recherche progresse différemment, selon une certaine circularité et sans qu'on puisse plus distinguer, de façon linéaire, les différentes étapes.

Quoiqu'il en soit, les sept étapes telles qu'elles sont expliquées et commentées par les auteurs donnent effectivement une idée claire de la démarche scientifique, et sans verser dans la simplification. Les auteurs insistent sur la notion de rupture avec les catégories ordinaires de l'entendement tout en discutant la 
question de la continuité entre le sens commun et la connaissance scientifique et rendent de façon très concrète la nécessité de construire le processus de recherche. La partie consacrée à la formulation de la question de départ n'est cependant pas la plus convaincante notamment en ce qu'elle tend à confondre en pratique la rupture avec le sens commun et la neutralité axiologique, alors même que le constructivisme est aussi largement revendiqué par une sociologie critique qui assume le rôle politique des sciences sociales. En ce sens, le travail sur la question de départ peut tout aussi efficacement servir au chercheur à prendre conscience de ses attentes normatives sans nécessairement en passer par une recherche de neutralité souvent artificielle. Par contre le chapitre sur l'exploration montre bien comment les lectures sont essentielles à l'entrée dans la recherche proprement dite et donnent d'utiles conseils sur la façon de les articuler avec des observations (entretiens, recueils d'informations de tous ordres) pour mieux définir le sujet de l'enquête. Le chapitre sur la problématique, dont les auteurs indiquent qu'il a été particulièrement retravaillé pour cette nouvelle édition, est effectivement très éclairant : les exemples sont mieux détaillés et surtout, les pages (90-98) destinées à montrer comment différents concepts construisent des projets fondamentalement différents à partir d'une même question semblent particulièrement réussies, notamment en qu'elles éclairent très simplement, et là aussi de façon fine, ce que peut signifier le caractère cumulatif de la recherche.

Le « panorama » des méthodes proposé, au stade de l'observation comme à celui de l'analyse, dépasse la seule opposition entre questionnaires et entretiens puisqu'il prend en compte notamment l'observation et l'analyse documentaire. Les présentations qui sont faites de chacune d'elles sont succinctes mais s'accompagnent de références bibliographiques introductives. $\mathrm{Au}$ total, en comptant ces bibliographies plus techniques, la bibliographie finale et les références insérées dans le texte lui-même, le Manuel s'appuie sur un ensemble de références tout à fait conséquent. Mais ce qui frappe, c'est qu'elles sont exclusivement en langue française. Etant donné le poids des travaux de langue anglaise dans le développement des sciences sociales, ce choix peut surprendre. Il n'est sans doute pas sans lien avec le fait que ces références sont également plutôt anciennes, dès lors que le volume de publications en langue française et d'ordre méthodologique, au sens large, au sens du Manuel, reste relativement limité.

Comme souvent, les pages consacrées à l'analyse des informations sont plutôt 


\section{On pourrait ajouter que tous les deux rythment les chapitres avec des exercices d'application qui, à défaut d'être vraiment utiles pour l'étudiant isolé, peuvent être repris et adaptés par les enseignants}

moins satisfaisantes, tant il est difficile de rendre compte de façon succincte et surtout, sans que le lecteur ait pu lui-même s’imprégner des données collectées, de la réalité du processus de décantation qui est au cœur du travail d'analyse. Peut-être d'ailleurs que les auteurs n'insistent pas suffisamment sur le temps qu'il faut pouvoir y consacrer puisqu'on sait combien, pour les étudiants, notamment dans le cas des mémoires de master, l'étape d'analyse intervient le plus souvent trop tard dans le processus de recherche.

Enquêtes qualitatives, enquêtes quantitatives et le Manuel de recherche en sciences sociales peuvent sembler complémentaires puisque le premier se concentre sur deux des sept étapes que détaille le second. Ils partagent d'ailleurs plusieurs traits communs : ils sont tous les deux le fruit d'une longue expérience d'enseignement qui les rend particulièrement accessibles, le Manuel de Campenhoudt et Quivy ayant en plus pour lui d'avoir su mettre à profit, au fil des éditions, les commentaires critiques de ses utilisateurs. La conception de la recherche qu'ils promeuvent est empirique, clairement centrée sur l'enquête. Et tous deux se limitent strictement à des références de langue française ${ }^{1}$. Pour autant, le message qu'ils véhiculent diffère sensiblement. Le livre dirigé par P.Bréchon, tout en revendiquant pour la recherche empirique une dimension de bricolage qui nécessite du chercheur, a minima, des capacités d'adaptation à la situation d'enquête, cherche à offrir à ses lecteurs une palette de solutions. La notion de « bonnes pratiques » à laquelle il se réfère est directement liée à un travail de standardisation et d'encadrement professionnels dont il faudrait interroger l'importation dans les sciences sociales. Le Manuel, lui, est plus tourné vers une explicitation de la démarche de recherche qui doit guider l'apprenti chercheur vers l'intelligibilité de sa propre pratique, susciter sa réflexivité.

On peut penser que cette seconde approche est mieux adaptée au contexte français. En effet, les sciences sociales françaises sont, par comparaison avec les sciences sociales de langue anglaise en particulier, beaucoup moins investies dans les discussions méthodologiques. Ceci est sans doute dû, en partie, aux dimensions réduites de notre communauté professionnelle, qui ne favorisent pas la spécialisation sur ce créneau dans un univers structuré par une spécialisation en paradigmes (ou écoles). Mais c'est aussi vraisemblablement le fait d'une approche très individualisée de la démarche scientifique qui, de plus, valorise largement l'apport théorique. Dès lors, on peut se réjouir du développement récent des échanges autour des questions de méthode mais 
y contribuer suppose d'adopter une position plus proche de celle de Quivy et Campenhoudt, dans laquelle les méthodes ne sont pas réductibles à des techniques encadrées par des règles, mais plutôt à des choix pratiques et circonstanciés dont l'amélioration est affaire d'inventivité et met essentiellement en jeu la réflexivité et l'échange. Ajoutons qu'il serait vraiment regrettable de limiter ces échanges au contexte francophone. 